\title{
The effects of healthy aging on intracerebral blood vessels visualized by magnetic resonance angiography
}

\author{
Elizabeth Bullitt, MD1, Donglin Zeng, PhD², Benedicte Mortamet ${ }^{3}$, Arpita Ghosh ${ }^{2}$, Stephen \\ R. Aylward, $\mathrm{PhD}^{4}$, Weili Lin, $\mathrm{PhD}^{5}$, Bonita L. Marks, $\mathbf{P h D}^{6}$, and Keith Smith, MD, $\mathbf{P h D}^{5}$ \\ ${ }^{1}$ CASILab, CB \#7062, Department of Surgery, University of North Carolina, Chapel Hill, NC, 27599 \\ ${ }^{2}$ Department of Biostatistics, University Of North Carolina, Chapel Hill, NC, $27599^{3}$ Ecole Advanced \\ Clinical Imaging Technology, Siemens Medical Solutions-CIBM, Lausanne, Switzerland ${ }^{4}$ Kitware, \\ Inc., Carrboro, NC $27510^{5}$ Department of Radiology, University of North Carolina, Chapel Hill, NC, \\ $27599{ }^{6}$ Department of Exercise and Sport Science, University of North Carolina at Chapel Hill, NC \\ 27599
}

\begin{abstract}
Histological and magnetic resonance imaging studies have demonstrated that age-associated alterations of the human brain may be at least partially related to vascular alterations. Relatively little information has been published on vascular changes associated with healthy aging, however. The study presented in this paper examined vessels segmented from standardized, high-resolution, magnetic resonance angiograms (MRA) of 100 healthy volunteers (50 males, 50 females), aged 18-74, without hypertension or other disease likely to affect the vasculature. The subject sample was divided into 5 age groups ( $n=20 /$ group) with gender equally distributed per group. The anterior cerebral, both middle cerebral, and the posterior circulations were examined for vessel number, vessel radius, and vessel tortuosity. Males exhibited larger vessel radii regardless of age and across all anatomical regions. Both males and females displayed a lower number of MRA-discernible vessels with age, most marked in the posterior circulation. Age-associated tortuosity increases were relatively mild. Our multi-modal image database has been made publicly available for use by other investigators.
\end{abstract}

\section{Keywords}

MRA; aging; vessels; brain; tortuosity

(C) 2008 Elsevier Inc. All rights reserved.

Corresponding author: Elizabeth Bullitt MD CASILab, CB \#7062, Department of Surgery, University of North Carolina, Chapel Hill, NC, 27599 (919) 843-3101 (office) (919) 643-1500 (fax) bullitt@ med.unc.edu.

Publisher's Disclaimer: This is a PDF file of an unedited manuscript that has been accepted for publication. As a service to our customers we are providing this early version of the manuscript. The manuscript will undergo copyediting, typesetting, and review of the resulting proof before it is published in its final citable form. Please note that during the production process errors may be discovered which could affect the content, and all legal disclaimers that apply to the journal pertain.

6. Disclosure statement for authors

The method of vessel segmentation has been patented and non-exclusively licensed to Medtronic (Minn, Minn), and R2 Technologies (Alta Vista, CA). Licensing agreements are under negotiation with WL Gore (Flagstaff, Arizona), and Kitware (Rochester, NY). The study described in this report was supported only by the NIH however; no commercial company contributed financial support. 


\section{Introduction}

The purpose of this report is to describe a new, noninvasive, in-vivo method of defining vascular changes associated with healthy aging and gender as depicted by magnetic resonance angiograms (MRA). Aging of the adult human brain is already known to be associated with a variety of anatomical tissue changes. Both post-mortem histological and in-vivo Magnetic Resonance (MR) imaging studies suggest that aging induces progressive shrinkage of grey matter volume (Courchesne et. al. 2000; Matsumae et. al. 1996; Peinado 1998; Resnick et. al. 2000). White matter is also affected, with an age-related decline in fractional anisotropy (Madden et. al. 2003; Sullivan and Pfefferrbaum 2006), loss of myelinated axons, (Marner et. al 2003) and alteration of white matter signals as seen by MR (Hachinski 1986). The variability is great, however, and the brains of some elderly subjects have the characteristics of the brains of much younger individuals (Creasey and Rapoport 2003).

One of the factors likely to contribute to the patient-specific severity of age-related anatomical and functional changes is the underlying status of the vasculature. Indeed, recent histological studies associate aging with plaque formation and microhemorrhage (Cullen et. al. 2006). Fernando et. al. (2006) report that the periventricular and deep subcortical white matter lesions commonly associated with advanced age are likely to arise as a result of chronic hypoperfusion, and Buckner (2004) also associates deep white matter lesions with microvascular disease. A recent histological analysis concludes that patients with deep white matter lesions exhibit capillary loss globally throughout the brain and not just only in the vicinity of the lesions. Furthermore, the decline in capillary number is age-related (Brown et al 2007). Life style choices may also affect the findings. Aerobic exercise training, which is likely to improve cardiovascular fitness, is associated with increased brain volume (Colcombe et al 2006). Similarly, Marks et. al. $(2007,2008)$ suggest that there may be a positive relationship between aerobic fitness and fractional anisotropy in select brain regions.

The majority of publications addressing the histology of the intracerebral vasculature have concentrated upon pathology. Relatively little direct information is available about genderassociated vascular differences or about the vessel changes that occur with aging in the absence of disease. By histological section, vessel tortuosity is known to increase with both aging and hypertension (Spangler et. al. 1994). Farkas et. al. (2006) note that microvascular pathology, such as fibrosis and vessel wall thickening, is age-related. Similarly, Uspenskaia et. al. (2004) describe reduction in the radius of microvessels in the elderly. Emerson et. al. (1994) however, find that cerebral perfusion appears to remain constant between the young (19-40 years old) and the elderly (65-85 years old). Takahashi et. al. (2005) note age-associated reductions of regional cerebral blood flow in the specific region of the anterior cingulate gyri, although these localized findings have been challenged on the basis of methods that fail to correct for partial volume effects (Pirson et. al. 2006). In potential contradiction to the findings of Emerson et. al. (1994), Zhao et. al. (2007) find an age-associated drop of about $15 \%$ in total cerebral and cranial blood flow in an analysis of 15 large, named arteries of younger (24-30 years, $n=12)$ and older $(61-74$ years, $n=4)$ subjects, as captured by phase contrast imaging.

In general, the literature on the vascular changes associated with healthy aging appears both sparse and sometimes contradictory. The current study employs a different approach to vascular assessment. We use computer-based methods to analyze in-vivo, time-of-flight, MRAs of 100 healthy volunteers, ranging in age from 18 to 74, and without history of hypertension, diabetes, head injury, or other factors likely to affect the cerebral vasculature. MR angiography is not capable of assessing vessels of diameter smaller than that of the voxel size used during image acquisition. This approach therefore cannot analyze capillaries. Nevertheless, this new method possesses unprecedented ability to provide quantitative, invivo, whole-head assessment of vessel number, radius, and tortuosity for all vessels perceptible 
by MRA and with analysis of the vasculature of the entire brain or of any desired portion of the brain.

The two hypotheses of the current study were that 1) healthy aging would be associated with change to intracerebral vessels visualized by MRA, and 2) there would be no difference between male and female subjects. This report is, to our knowledge, the first to explore genderassociated changes in vessel shape and number with healthy aging as perceived by MRA.

\section{Methods}

\subsection{Patient selection and image acquisition}

One hundred healthy volunteers, divided into five age groups, were involved in this study. Each age group (18-29, 30-39, 40-49, 50-59, and 60-74 years) comprised 20 subjects equally divided by gender. Subjects with hypertension, diabetes, hypercholesterolemia, coagulopathy, mental illness, vascular disease, or brain disorder were excluded. The study was approved by the Institutional Review Board of the University of North Carolina at Chapel Hill, was compliant with the United States' Health Insurance Portabiliity and Accountability Act (HIPAA), and all subjects provided signed consent to share their images with other investigators.

All volunteers underwent MR scanning on a 3T, head only unit (Allegra, Siemens Medical Systems Inc.) using a head coil. T1, T2, DTI, and MRA images were acquired for each subject. This paper concentrates upon vascular images. MRA images were acquired using a 3D-timeof-flight sequence without gadolinium injection and that covered the entire head using multiple (5) overlapping (25\%) thin slabs (MOTSA). The sequence also employed a magnetization transfer pulse for background suppression of brain parenchyma. The resulting voxel size was $0.5 \times 0.5 \times 0.8 \mathrm{~mm}^{3}$. The sequence's TR/TE/flip_angle/ were respectively $35 \mathrm{msec} / 3 \mathrm{msec} / 22$. Although the base matrix was $448 \times 448$, a rectangular FOV of 0.786 and a partial Fourier of 0.8 were employed to reduce data acquisition time to 18 minutes.

As discussed later, a secondary study additionally made use of the subjects' T1 and T2 images. Imaging parameters for these acquisitions included: TR $/ \mathrm{TE} / \mathrm{TH} /=15 \mathrm{msec} / 7 \mathrm{msec} / 1 \mathrm{~mm}$ for $\mathrm{T} 1$ and TR/TE$/ \mathrm{TH}=7730 \mathrm{msec} / 80 \mathrm{msec} / 1 \mathrm{~mm}$ for T2 . Both T1 and T2 images covered the entire brain and employed a voxel size of $1 \times 1 \times 1 \mathrm{~mm}^{3}$. Both sequences were acquired in $3 \mathrm{D}$ rather than in the more typically acquired $2 \mathrm{D}$ format.

\subsection{Image processing}

Image processing methods employed $\mathrm{C}++$ programs written by our group and previously described in the literature. Vessels were segmented using a computer program that, proceeding from manually provided seed points, automatically tracked each vessel's central skeleton in three dimensions (3D) as an image intensity ridge using dynamic scaling (Aylward et. al. 2002). The vessel radius was then calculated automatically at each skeleton point, with automatic smoothing of the skeleton points and radii using a spline function (Aylward et. al. 2002). Vessel skeleton points (with associated radius values) were subsequently resampled using a spacing equal to that of the voxel spacing in the $\mathrm{x}$ dimension of the underlying image $(0.5 \mathrm{~mm}$ in this study).

Following vessel segmentation, 3D vessel trees were defined via a computer program that requested manual definition of one or more roots (in this case, both carotid arteries and the basilar artery) and that then automatically defined connected vessel trees using a minimum spanning tree algorithm (Bullitt et al 2001). Blood flow direction was set automatically by the program during connection of each child vessel to a parent vessel. It took approximately 30 minutes to segment and create vessel trees from each MRA image. 
In this paper, we use the term "subtree" to designate the set of connected vessels that receive blood flow from (i.e., lie downstream of) a manually selected vessel point. Determination of vessel attributes was performed using a computer program that, via a 3D display, allowed "point and click" operations to manually designate vessel points of interest and to thus define subtrees of interest.

Four different anatomical regions were defined for each subject. Manually placed vessel branch points, selected to define major branches that could be easily identified across subjects, were used to define the origins of subtrees of interest. Specifically, the identified branch points were the origin of the left and right M1 segments, the paired A1 segments, and the distal basilar artery immediately before bifurcation into the left and right posterior cerebral arteries. Each image therefore provided 4 sets of connected vessels from four different anatomical regions each of which could be identified in all patients. These regions comprised the left middle cerebral group, the right middle cerebral group, the anterior cerebral group, and the posterior cerebral circulation. The anterior cerebral group contained two subtrees (one associated with each of the paired anterior cerebral arteries) that were analyzed together as a single unit.

Four different vessel attributes were analyzed for each vessel group. These attributes were

1. Vessel Number: The number of individual, unbranched vessels.

2. Average Radius: The sum of radii at all vessel points divided by the number of vessel points.

3. Two Tortuosity Measurements:

a. The "Sum of Angles Metric" (SOAM). SOAM sums angles between consecutive trios of points along a regularly sampled space curve and normalizes by the total path length (Bullitt et al 2003). Space curves displaying low-frequency, high amplitude curves tend to display high SOAM values. The average SOAM value was calculated for each vessel group.

b. The "Inflection Count Metric" (ICM). The ICM counts the number of inflection points along a space curve and multiples this number (plus 1) times the total path length and divides by the distance between endpoints (Bullitt et. al. 2003). Space curves that exhibit high amplitude curves with frequent changes of direction will tend to display high ICM values. The average ICM value was calculated for each vessel group.

\subsection{Statistical analysis}

Analysis was performed for each of the four vessel groups both by gender and age. A preliminary test using Kolmogrov-Smirnov statistics was performed to test for normality of the values, and was followed by Tukey tests for overall comparisons of the four parameters across anatomical groups. To examine the pattern of the four measures by age and gender, a multivariate ANOVA was fit to the data. The within-subject factors included the four types of measurements and the four regions. The between-subject factors included the five age groups and two genders. Interaction between age and gender was included in the model. To adjust for multiple comparisons of four measures, $0.05 / 4$ was used as the significance level. For each measure, the comparison across different factor levels was based on the Tukey test. All analyses were done using SAS 8.0 [SAS Institute, Rearch Triangle Park, North Carolina]. 


\subsection{Secondary analyses}

Results of the primary study outlined above produced two findings that deserved further exploration. One of these findings was related to vessel radius. It became of interest to determine the average vessel radius for large, individual, named arteries.

Although the carotid and basilar arteries were obvious candidates for this secondary study, our images displayed variability in the lowest anatomical region imaged. Long lengths of the carotid and basilar arteries were therefore available for study in some volunteers but not in others. In order to standardize the vessel segments analyzed, we therefore selected 5 large vessels in which proximal and distal vessel points could be reliably determined across all subjects. The five arterial segments chosen for analysis included the left and right A1 segments, the left and right M1 segments, and the segment of the basilar artery between the takeoff of the superior cerebellar arteries and the basilar tip. Each defining vessel branchpoint was selected manually using a 3D, rotatable display (Bullitt et. al., 2001). The program clipped each vessel proximally and distally to the manually selected points and then calculated vessel attributes for the selected vessel segment. Multivariate ANOVA was used to analyze the outcomes.

A second finding deserving further exploration was related to vessel count. Since brain volume differs from individual to individual, it became of interest to normalize vessel counts for each individual by that individual's volume of grey and white matter. We determined the volumes of grey matter, white matter, and CSF from each individual's T1 and T2 images using a segmentation algorithm based upon the expectation-maximization algorithm developed by Van Leemput et. al. (1999a, 1999b) and as rewritten as an integrated package in ITK (Insight Toolkit). The program automatically registered the $\mathrm{T} 1$ and $\mathrm{T} 2$ images of each patient, performed bias field inhomogeneity correction, registered the image pair to a spatial probabilistic brain atlas (Evans 1993), and finally calculated probabilistic maps for grey matter, white matter, and CSF (Mortamet 2005). Brain volume was calculated by integration over grey and white matter regions for each patient. We then calculated the total vessel count in each subject and divided that count by that subject's brain volume. We did not perform similar analyses for each of the four circulatory groups examined in our primary analysis since we did not have a means of reliably standardizing the tissue boundaries perfused by each circulatory group. Statistical analysis was based on fitting ANOVA model, where the outcome was the normalized vessel count and two factors were Age groups (5 groups) and gender.

\section{Results}

\subsection{Results of the primary analysis}

Figure 1 illustrates the 4 vessel groups shown in 3D for one of the healthy volunteers. Figure 2 illustrates a typical MRA slice with and without a superimposed red overlay of the segmented vessels within that slice. Figures 3-6 provide the means and standard deviations calculated for vessel number, average vessel radius, and the two tortuosity measures SOAM and ICM for each of the five age groups, for each gender, and for each of the four anatomical regions analyzed.

3.1.1 Vessel attributes by anatomical location-When the 100 healthy subjects were considered as a group, all four vessel attributes differed significantly by anatomical region ( $\mathrm{p}$ values were $<0.0001$ for vessel count, $<0.0001$ for SOAM, 0.0206 for AVRAD and $<0.001$ for ICM).

Vessel Number: Vessel number in the anterior cerebral group was less than in the left middle cerebral distribution ( $\mathrm{p}=0.0032$ ); vessel numbers in the left and right middle cerebral 
distributions did not differ significantly; vessel numbers in the posterior circulation were significantly greater than in any other distribution $(\mathrm{p}<0.001)$. Means and standard deviations for vessel number are shown in Figure 3.

Average Radius: A significant difference $(\mathrm{p}=0.0147)$ was observed between the anterior and the left middle cerebral circulations. Means and standard deviations for radius are shown in Figure 4.

Tortuosity: SOAM values differed significantly across anatomical regions $(\mathrm{p}<0.0001)$. The posterior circulation possessed the highest values, the anterior cerebral SOAM was less than the left middle cerebral SOAM, and no significant differences were observed between the left and right middle cerebral SOAMs . For the ICM, the left middle cerebral circulation had significantly smaller ICM than the right middle cerebral circulation $(\mathrm{p}=0.002)$ and significantly larger ICM than the posterior circulation $(\mathrm{p}<0.0001)$. No significant difference was detected between the anterior and left middle cerebral groups. Means and standard deviations for SOAM and ICM calculations are shown in Figures 5 and 6.

3.1.2 Gender and age comparisons-Major findings for the gender and age comparisons are summarized below. Graphical representations of the means and standard deviations for each vessel parameter are given in Figures 3-6. For purposes of brevity, we use the term "vessel loss" to indicate a reduction in the number of vessels visualized by MRA. As discussed later, a reduction in the number of vessels visualized by MRA could represent an absolute reduction in the number of vessels present, a reduction in blood flow (thus making some vessels invisible to MRA although the vessels themselves might remain physically present), or both.

1) Vessel Number: Both genders exhibited a trend toward vessel loss with age in all 4 anatomical regions. The vessel count in the left middle cerebral circulation was significantly lower in subjects aged 60+ than in those aged 18-29 ( $\mathrm{p}=0.002)$. Results were most marked in the posterior circulation, for which the vessel count was significantly lower in those aged 60+ than in any other age group (all p-values less than 0.0025).

2) Average Radius: In all four anatomical regions, males exhibited vessels of larger average radius than did women (anterior cerebral group. $\mathrm{p}=0.0021$; left middle cerebral group, $\mathrm{p}=0.0006$; right middle cerebral group, $\mathrm{p}=0.0002$; posterior cerebral group, $\mathrm{p}=0.0033$ ). Aging was also associated with increase in average radius. For those aged $60+$, the average radius of the right middle cerebral group was larger than that of subjects aged $18-29(\mathrm{p}=0.0008)$, as was the average radius of the posterior cerebral circulation $(\mathrm{p}=0.0058)$. No significant interaction between gender and age was found.

1. SOAM. Significant changes with aging were limited to the left middle cerebral distribution. Subjects aged $60+$ exhibited a significant increase in tortuosity by SOAM compared to those 18-29 ( $\mathrm{p}=0.0118)$ and 30-39 ( $\mathrm{p}=0.0025)$.

2. ICM. There was no significant difference between age groups or genders in any region.

\subsection{Secondary analysis of vessel radius in large named vessels}

Figure 7 summarizes the results of radius calculation in each of the five age groups for five major named vessels. Males consistently displayed higher average vessel radii than females. This difference was present in all five of the individual vessels analyzed and across all age groups (p-values all less than 0.02). When assessing the effect of aging upon the radius of the large named vessels, the older age groups tended to display larger vessel radii than the younger age groups. The difference was most consistently evident in the right M1 arterial segment 
$(\mathrm{p}=0.015)$. More specifically, for RM1, age group 1 possessed smaller vessel radii than age group 5 ( $\mathrm{p}=0.042$ ); age group2 had smaller vessel radii than age group 3 ( $\mathrm{p}=0.047)$; age group 2 had smaller vessel radii than age group $5(\mathrm{p}=0.002)$; age group 4 had smaller vessel radii than age group $5(\mathrm{p}=0.007)$.

\subsection{Secondary analysis of normalized vessel count}

Figure 8 provides the results of total vessel count normalized by brain volume. Females tended to have significantly larger values than males $(\mathrm{p}<0.0001)$. We did not have enough power to discriminate between the first three younger age groups or the last two older age groups. However, after combining the first three groups into one group and combining the last two groups into another, results indicated that the younger age group had significantly higher normalized vessel counts than the older $(\mathrm{p}=0.0005)$.

\section{Discussion}

\subsection{General methodological considerations and comparison to other approaches}

This report is, to our knowledge, the first to evaluate the effects of gender and healthy aging upon intracerebral vessel number and morphology as visualized by non-invasively acquired MRA. Our approach offers a method complementary to that of other published approaches and possesses different strengths and weaknesses. Unlike histological analyses, our method cannot assess capillaries. It can, however, rapidly and noninvasively assess the vasculature of the entire brain as captured by MRA. A related advantage of our method, although not applicable to the current study, is that our approach is capable of following changes to an individual patient over time--clearly impossible for whole-brain histological processing.

A variety of in-vivo vascular imaging methods have been described by others. The method that is perhaps most similar to our own is outlined by Zhao et. al. (2007), who used phase contrast imaging to calculate regional cerebral blood flow based upon measurements from 15 major vessels. Our own approach only approximates regional cerebral blood flow on the basis of vessel radius and number. By contrast, unlike the methods described by Zhao et. al. (2007), our approach (a) is not restricted to large, named vessels but can evaluate many hundreds of vessels including even the smallest vessels perceptible by MRA, and (b) provides quantitative measures of vessel number, radius, and tortuosity.

The most commonly employed method of noninvasive, in-vivo vascular imaging of brain is perfusion/permeability imaging. Unlike perfusion/permeability imaging, our method cannot assess the capillary bed, but does offer the advantage of providing specific, quantitative information about vessel number, radius, and tortuosity in a way that perfusion/permeability imaging cannot. Bullitt (2007) provides an overview of the advantages and disadvantages of perfusion/permeability and MRA imaging as applied to tumor-associated vasculature. The two methods appear to provide complementary information.

Our methods provide radius estimates for large named vessels that are somewhat smaller than those provided by cadaveric dissection. For example, our estimate of the average diameter of the A1 segment was slightly over $1.5 \mathrm{~mm}$ whereas Rhoton reports that that the A1 segment is $1.5 \mathrm{~mm}$ or less in diameter in only $10 \%$ of brains [Rhoton, 2003]. There are at least two explanations for this discrepancy. The first is that our approach employs MRA images that visualize flowing blood. As a result, our calculation of vessel radius does not include the thickness of the vessel wall. Our method of calculating vessel radius will thus obviously be smaller than any method that does include the vessel wall. The second explanation is related to our method of vessel extraction. Our method employs adaptive scaling [Aylward et. al., 2002] and is thus able to define a single, long vessel as possessing a large radius proximally 
and a small radius distally. MRA images are noisy, however, and in order to prevent errors when regions of image noise are encountered during vessel extraction, the radius calculated for any individual point is based partially upon the specific value calculated for that particular point, partially upon the radii calculated for sets of vessel points proximal and distal to the point in question, and partially upon an initial estimate of the overall vessel's radius. As a result, the radius of a long vessel may sometimes be underestimated in regions in which the radius is large and overestimated in regions in which the radius is small. Although the difference is generally slight between calculated radius and the radius as perceived by inspection of slice data (see Figure 2, for example), this difference, in combination with the fact that MRA images do not visualize the vessel wall, seems likely to explain the reason why our calculation of the radii of large named vessels is slightly smaller than calculations based upon histological or cadaveric analysis.

We believe that a major contribution of our report is that it describes the use of high-resolution $\left(0.5 \times 0.5 \times 0.8 \mathrm{~mm}^{3}\right)$, standardized images acquired from 100 volunteers equally divided into 5 different age groups each equally divided by gender. We also carefully screened our volunteers for the absence of hypertension, diabetes, and other diseases likely to affect the vasculature. The majority of previous publications on vessel changes associated with aging have included subjects with a variety of age-associated diseases, and the majority of in-vivo imaging studies have used either varying protocols and/or large or grossly anisotropic voxels.

Our gender and age-matched healthy database of high-quality MRA, T1, T2, and DTI images all acquired in standardized fashion on a $3 \mathrm{~T}$ are freely available to the scientific community at http://hdl.handle.net/1926/594.

\subsection{Discussion of specific findings}

At least six of our specific findings deserve comment.

First, there is no single set of vessel descriptors that defines the shape of a prototypical healthy intracerebral vessel independent of anatomical location. As shown by the results of our study, the shapes of healthy intracerebral vessels differ by anatomical location. The implication of this finding is that any analysis that compares patient groups must compare similar regions of the brain or similar vascular structures across groups rather than compare any test vessel to some absolute, abstract definition of what a "healthy" intracerebral vessel should be.

Second, the current study analyzed 4 independent, anatomically separated vascular subtrees, each of which was identifiable across all healthy volunteers. An equally valid approach when addressing focal disease is to clip vessels to the same anatomical region of interest and to analyze vessels within the same region across subjects (Bullitt 2005). A number of equally valid approaches also exist (analysis of vessels of similar radii, analysis of individual named vessels) that are applicable to other disease states, but discussion of the choice of which analysis is likely to be most helpful when assessing any particular disease state is beyond the scope of this paper. Our approach to vessel segmentation and vessel shape analysis permits all of these approaches, with the choice of the approach open to the investigator.

Third, an unexpected finding of our study was that males and females exhibit consistent differences in vessel radius when vessels are defined from MRA. Contrary to our initial hypothesis of no gender-specific vascular differences, this study found that males exhibited significantly larger vessel radii than females in all four anatomical regions, independent of age. This difference remained present no matter whether entire circulatory groups were analyzed or whether specific, large, named vessels were identified across individuals. It is possible that these differences may be related to the larger cerebral volume of males (Matsumae et. al. 1996; Resnick et. al. 2003) who then might require feeding arterial trunks of larger radius to 
supply a more extensive capillary network. The work by Zhao et. al. (2007) noted that flow through the carotid arteries was significantly larger in males than in females, further supporting the validity of our findings.

Fourth, healthy aging was associated with a gender-independent trend towards reduction in the number of vessels visualized by MRA. This trend persisted even when the total vessel count for each individual was normalized by that individual's brain volume. The reduction in vessel number with age thus cannot easily be explained on the basis of brain atrophy alone. One explanation for this finding is an age-related, physical loss of vessels. This interpretation is supported by some histological analyses (Brown 2007; Peters 2006; Srinkath 2003) although it is difficult to find a histological report that quantifies vessel loss in the absence of disease. An alternative explanation is an age-associated reduction to blood flow, which could render vessels invisible to MRA although the vessels themselves might remain physically present. In this case, our findings would represent functional changes (underperfused vessels) rather than anatomical changes. Indeed, the groups of Farkas et. al. (2006) and Uspenskaia et. al. (2004) note that the thickness of the vessel wall increases and that the radius of microvessels decreases with age. Although our methods cannot visualize microvessels, it is possible that aging induces thickening of the vessel walls of the smaller vessels visualizable by MRA. It is moreover possible that both factors (physical loss of vessels and reduced blood flow) played a role in our results. Regardless of the interpretation (physical vessel loss, reduced blood flow, or both), our findings indicate that aged subjects imaged by the same protocols as younger subjects exhibit a lower number of visualizable vessels, indicating a change in vessel attributes with healthy aging.

A fifth interesting finding was the increase in average vessel radius that occurred with healthy aging. There are at least two explanations for this finding. The first is that there was a loss (or a reduction in perfusion resulting in a lack of visualization) of the smaller vessels visualized by MRA. A decrease in the number of these smaller vessels would produce an increase in the average vessel radius. The second explanation is that there was a compensatory dilation of proximal vessels. In an attempt to begin to answer this question we examined the radii of five proximal, major named vessels. The finding that average radius tended to increase with age in these proximal vessels suggests that compensatory vessel dilation may play a role in the increased vessel radius seen with healthy aging.

The sixth and last finding was that a significant increase in vessel tortuosity with age was limited to the left middle cerebral distribution and with tortuosity as measured only by SOAM. No significant changes with age were seen by tortuosity as measured by ICM in any anatomical distribution. Histological and other types of examinations have indicated that significant increases in vessel tortuosity are associated with a variety of diseases including hypertension (Hiroki et. al. 2002; Spangler 1994), Alzheimer's Disease (Fischer et. al. 1989; Kalaria 2002), cancer (Baish and Jain 2000; Bullitt et. al. 2005; Jain 2005; Li et. al. 2000) and many other pathological processes (Burger et. al. 1991). Our results suggest that the vessel tortuosity increases that occur with healthy aging are relatively modest.

\subsection{Study limitations}

Three limitations of the current study should be noted. The first was that the most aged group was $60+$ and that the most elderly subject in the database was 74 . We therefore did not examine patients 80 years or older, nor did we have many patients older than 70 . The reason is that it becomes progressively difficult to identify subjects without hyptertension, diabetes, hypercholesterolemia, renal disease, or other history of disease likely to affect the cerebral vasculature once individuals reach the age of 60 . Our intent in acquiring this database was to provide an image database of genuinely healthy volunteers that could be used both to assess the effects of healthy aging and, perhaps more importantly, to establish an image database of 
healthy volunteers against which a variety of diseases can be assessed. Although it should be feasible to accumulate a sufficient number of healthy subjects in their 70s and 80s and older, such recruitment would require much more time and funding than was available to us. Our current database therefore sets the highest age group as $60+$.

A second limitation of our approach was that MRA imaging inherently cannot examine any vessel of diameter smaller than that of the voxel size used during image acquisition. For the current study, vessel analysis was thus limited to vessels of $0.25 \mathrm{~mm}$ radius or larger. A contribution of this study is the finding that noninvasive analysis of these "larger" vessels over a more extensive anatomical region than that provided by traditional histological processing can produce similar results, demonstrating a spatially widespread dropout of vessel number and/or reduction in functional capacity with age.

A final limitation of our approach was that we did not control for multiple factors including diet, activity level, and perhaps many other factors of whose importance we may be unaware. Several recent reports have suggested that activity level may affect the cerebrovasculature, brain volume, and cognitive level during healthy aging (Colcombe et. al. 2004, Marks et. al. 2000). More directed studies aimed at examining individual factors would be required to assess the importance of each such parameter.

\subsection{Summary}

In summary, this study provides an analysis of MRA images acquired in-vivo of 100 healthy subjects aged 18-74. We use a designed database in which subjects were excluded for known disease, in which all images were acquired similarly and at high resolution, and in which equal numbers of males and females were included in each of five age groups. We conclude that there are differences between the vasculature of males and females, that healthy aging is associated with both a loss of vessels and an increase in vessel radius for those vessels visualized by MRA, and that any vessel tortuosity increases associated with healthy aging are relatively modest. Our new approach offers information complementary to that provided by other methods. Our multi-modal image database has been made publicly available to the scientific community. We believe that this database could serve as an important resource not only for an analysis of healthy aging but also as a healthy baseline against which to compare a variety of vessel abnormalities associated with multiple pathological states.

\section{Acknowledgments}

Supported by R01EB000219 NIH-NIBIB

\section{Reference List}

Aylward SR, Bullitt E. Initialization, noise, singularities and scale in height ridge traversal for tubular object centerline extraction. IEEE-TMI 2002;21:61-75.

Baish JS, Jain RK. Fractals and cancer. Cancer Research 2000;60:3683-3688. [PubMed: 10919633]

Brown WR, Moody DM, Thore CR, Challa VR, Anstrom JA. Vascular dementia in leuokoaraiosis may be a consequence of capillary loss not only in the lesions, but in normal-appearing white matter and cortex as well. J Neurol Sci 2007;257:62-66. [PubMed: 17320909]

Buckner RL. Memory and executive function in aging and AD: decline and reserve factors that compensate. Neuron 2004;44:195-208. [PubMed: 15450170]

Burger, PC.; Scheithauer, BW.; Vogel, FS. Surgical Pathology of the Nervous System and its Coverings. Vol. Third Edition. Churchill Livingstone; New York: 1991.

Bullitt E, Aylward S, Smith K, Mukherji S, Jiroutek M, Muller K. Symbolic Description of Intracerebral Vessels Segmented from MRA and Evaluation by Comparison with X-Ray Angiograms. Medical Image Analysis 2001;5:157-169. [PubMed: 11516709] 
Bullitt E, Gerig G, Pizer S, Aylward SR. Measuring tortuosity of the intracerebral vasculature from MRA images. IEEE-TMI 2003;22:1163-1171.

Bullitt E, Zeng D, Gerig G, Aylward S, Joshi S, Smith JK, Lin W, Ewend MG. Vessel tortuosity and brain tumor malignancy: A blinded study. Academic Radiology 2005;12:1232-1240. [PubMed: 16179200]

Bullitt E, Reardon DA, Smith JS. A Review of Micro and Macro Vascular Analyses in the Assessment of Tumor-Associated Vasculature as Visualized by MR. NeuroImage 2007;37(Supp 1):116-119. [PubMed: 17543543]

Colcombe SJ, Erickson KI, Scalf PE, Kim JS, Prakash R, McAuley E, Elavsky S, Marquez DX, Hu L, Kramer AF. Aerobic exercise training increases brain volume in aging humans. The Journals of Gerontology Series A: Biological Sciences and Medical Sciences 2006;61:1166-1170.

Courchesne E, Chisum HJ, Townsend J, Cowles A, Covington J, Egaas B, Harwood M, Hinds S, Press GA. Normal brain development and aging: quantitative analysis at in vivo MR imaging in healthy volunteers. Radiology 2000;216:672-682. [PubMed: 10966694]

Creasey H, Rapoport SI. The aging human brain. Annals of Neurology 2003;17:2-10. [PubMed: 3885841]

Cullen KM, Kocsi Z, Stone J. Microvascular pathology in the aging human brain: evidence that senile plaques are sites of microhaemorrhages. Neurobiol Aging 2006;27:1786-966. [PubMed: 17063559]

Emerson JF, Chen PC, Shankle WR, Haier RJ, Nalcioglu O. Data analysis for dynamic constrastenhanced MRI-based cerebral perfusion measurements: correcting for changing cortical CSF volumes. Magnetic Resonance Materials in Physics, Biology, and Medicine 1994;3:41-48.

Evans, AC.; Collins, DL.; Mills, SR.; Brown, ED.; Kelly, RL.; Peters, TM. 3D statistical neuroanatomical models from 305 MRI volumes.. Proc. IEEE Nuclear Science Symposium and Medical Imaging Conference; 1993. p. 1813-1817.

Farkas E, de Vos RAI, Donka G, Steur ENJ, Mihaly A, Luiten PGM. Age-related microvascular degeneration in the human cerebral periventricular white matter. Acta Neuropath 2006;111:150-157. [PubMed: 16453142]

Fernando M, Simpson JE, Matthews F, Brayne C, Lewis CE, Barber R, Kalaria RN, Forster G, Esteves F, Wharton S, Shaw PJ, O'Brien JT, Ince PG. White matter lesions in an unselected cohort of the elderly: molecular pathology suggests origin from chronic hypoperfusion injury. Stroke 2006;37:1391-1398. [PubMed: 16627790]

Fischer VW, Siddiqi A, Yusufaly Y. Altered angioarchitecture in selected areas of brains with Alzheimer's disease. Acta Neuropathologica 1990;79:672-679. [PubMed: 2360411]

Hachinski VC, Potter P, Merskey H. Leuko-araiosis: An ancient term for a new problem. Can J Neurol Sci 1986;13(4 Suppl):533-4. [PubMed: 3791068]

Hiroki M, Miyashita K, Oda M. Tortuosity of the white matter medullary arteries is related to the severity of hypertension. Cerebrovascular Diseases 2002;13:242-250. [PubMed: 12011548]

Jain RK. Normalization of the tumor vasculature: An emerging concept in anti-angiogenic therapy. Science 2005;307:58-62. [PubMed: 15637262]

Kalaria RN. Small vessel disease and Alzheimer's dementia: pathological considerations. Cerebrovascular Diseases 2002;13(Suppl 2):48-52. [PubMed: 11901243]

Li CH, Shan S, Huang Q, Braun RD, Lanzen J, Hu K, Lin P, Dewhirst MW. Initial stages of tumor cellinduced angiogenesis: evaluation via skin window chambers in rodent models. J Natl Cancer Inst 2000;92:143-147. [PubMed: 10639516]

Marks BL, Katz LM, Nunley DC, Neelon V, Daniel P. Cerebral blood flow and ccognitive function is maintained in aerobically active older adults. Circulation, Suppl 2000;102(18):II-875.

Marks, BL.; Madden, DJ.; Bucur, B.; Provenzale, JM.; White, LE.; Cabeza, R.; Huettel, SA. Role of aerobic fitness and aging on cerebral white matter integrity.. In: de Leon, MJ.; Snider, DA.; Federoff, H., editors. Imaging and the Aging Brain. Vol. 1097. Annals of the New York Academy of Science; 2007. p. 171-174.

Marks BL, Katz LM, Styner M, Smith JK, Babbitt M, Hawkins J, Bullitt E. Aerobic fitness impact on cerebral white matter integrity in the cingulum. Medicine and Science in Sports and Exercise 2008;40:5. Supplement (May). 
Madden DJ, Whiting WL, Huettel SA, White LE, MacFall JR, Provenzale JM. Diffusion tensor imaging of adult age differences in cerebral white matter: Relation to response time. NeuroImage 2004;21:1174-1181. [PubMed: 15006684]

Marner L, Nyengaard JR, Tang Y, Pakkenberg B. Marked loss of myelinated nerve fibers in the human brain with age. J Comp Neurol 2003;462:144-152. [PubMed: 12794739]

Matsumae M, Kikinis R, Mórocz I, Lorenzo A, Sándor T, Albert MS, McL. Black P, Jolesz F. Age related changes in intracranial compartment volumes in normal adults assessed by MRI. J Neurosurg 1996;84 (6):982-91. [PubMed: 8847593]

Mortamet B, Zeng D, Gerig G, Prastawa M, Bullitt E. Effects of healthy aging measured by intracranial compartment volumes using a designed MR database. Lecture Notes in Computer Science 2005;3749:383-391.

Peinado MA. Histology and histochemistry of the aging cerebral cortex: an overview. Microscopy Research and Technique 1998;43:1-7. [PubMed: 9829452]

Peters R. Ageing and the brain. Postgraduate Medical Journal 2006;82:84-88. [PubMed: 16461469]

Pirson AS, Borght TV, Van Laere K. Age and gender effects on normal regional cerebral blood flow. AJNR 2006;27:1161-1163. [PubMed: 16775255]

Resnick SM, Pham DL, Kraut MA, Zonderman AB, Davatzikos C. Longitudinal magnetic resonance imaging studies of older adults: a shrinking brain. J Neurosci 2003;23(8):3295-301. [PubMed: 12716936]

Rhoton, AL. Cranial Anatomy and Surgical Approaches. Lippincott, Williams and Wilkins; Schaumburg, Ill: 2003.

Spangler KM, Chandra VR, Moody DM. Arteriolar tortuosity of the white matter in aging and hypertension. A microradiographic study. J Neuropathol Exp Neurol 1994;53:22-26. [PubMed: 8301316]

Srinkath, VK.; Donnan, GA. Chapter 9. Cerebrovascular system and the ageing brain.. In: Sachdev, PS., editor. The Neurobiology and Neuropsychiatry of Ageing. Swets and Zeitlinger; Netherlands: 2003.

Sullivan EV, Pfefferbaum A. Diffusion tensor imaging and aging. Neuroscience and Biobehavioral Reviews 2006;30:759-761.

Takahashi K, Yamaguchi S, Kobayasu S, Yamamoto Y. Effects of aging on regional cerebral blood flow assessed by using technetium Tc 99m hexamethylpropyleneamine oxime single-photon emission tomography with 3D stereotactic surface projection analysis. AJNR 2005;26:2005-2009. [PubMed: 16155150]

Uspenskaia O, Liebetrau M, Herms J, Danek A, Hamann GF. Aging is associated with increased collagen type IV accumulation in the basal lamina of human cerebral microvessels. BMC Neuroscience 2004;5:37-43. [PubMed: 15387892]

Van Leemput K, Maes F, Vandermeulen D, Suetens P. Automated model based tissue classification of MR images of the brain. IEEE Trans Med Imaging 1999a;18:897-908. [PubMed: 10628949]

Van Leemput K, Maes F, Vandermeulen D, Suetens P. Automated model based bias field correction of MR images of the brain. IEEE Trans Med Imaging 1999b;18:885-896. [PubMed: 10628948]

Zhao M, Amin-Hanjani S, Ruland S, Curcio AP, Ostergren L, Charbel FT. Regional cerebral blood flow using quantitative MR angiography. AJNR 2007;28:1470-1473. [PubMed: 17846193] 

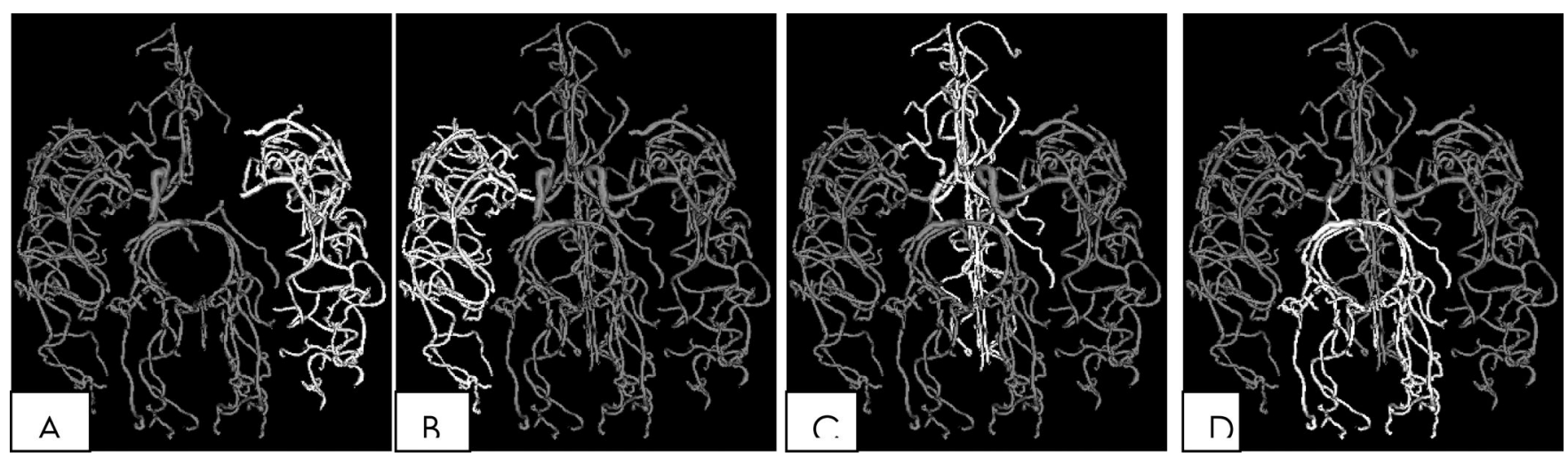

Figure 1.

Base view of a 3D visualization of a vessel segmentation illustrating the four vessel groups analyzed. The pertinent vessel group is shown in white in each frame. A: left middle cerebral, $\mathrm{B}$ : right middle cerebral, $\mathrm{C}$ : anterior cerebral, and D: posterior cerebral groups. 


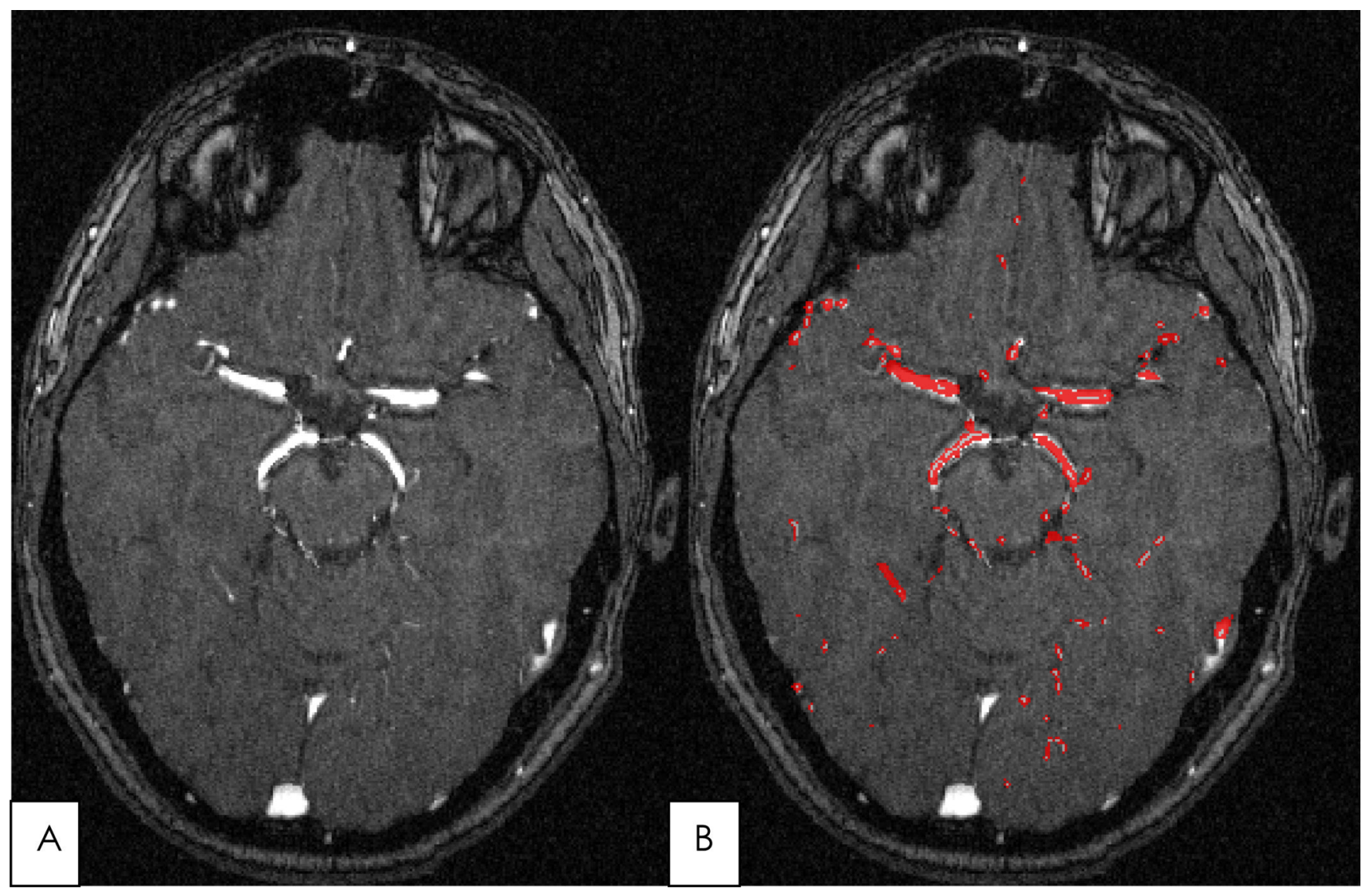

Figure 2.

Segmented vessels superimposed upon an image slice. A. Original slice through an MR image. B. The same slice with segmented vessels within that slice displayed in red. The images include both large arteries (the M1 segments bilaterally and the posterior cerebral arteries bilaterally) and small vessels, including a few that are only a voxel in diameter. Obvious veins, such as the sagittal and straight sinuses, are not included in the segmentation and remain white in panel B. When segmented vessels are overlaid in red upon the original MRA slice (B), the diameters of the segmented vessels seem to match reasonably well to the diameters of the vessels as visualized by the original MRA, although large vessels may be slightly underfilled.

Underfilling can be perceived as residual white voxels within a vessel whose segmentation is demarcated in red. 


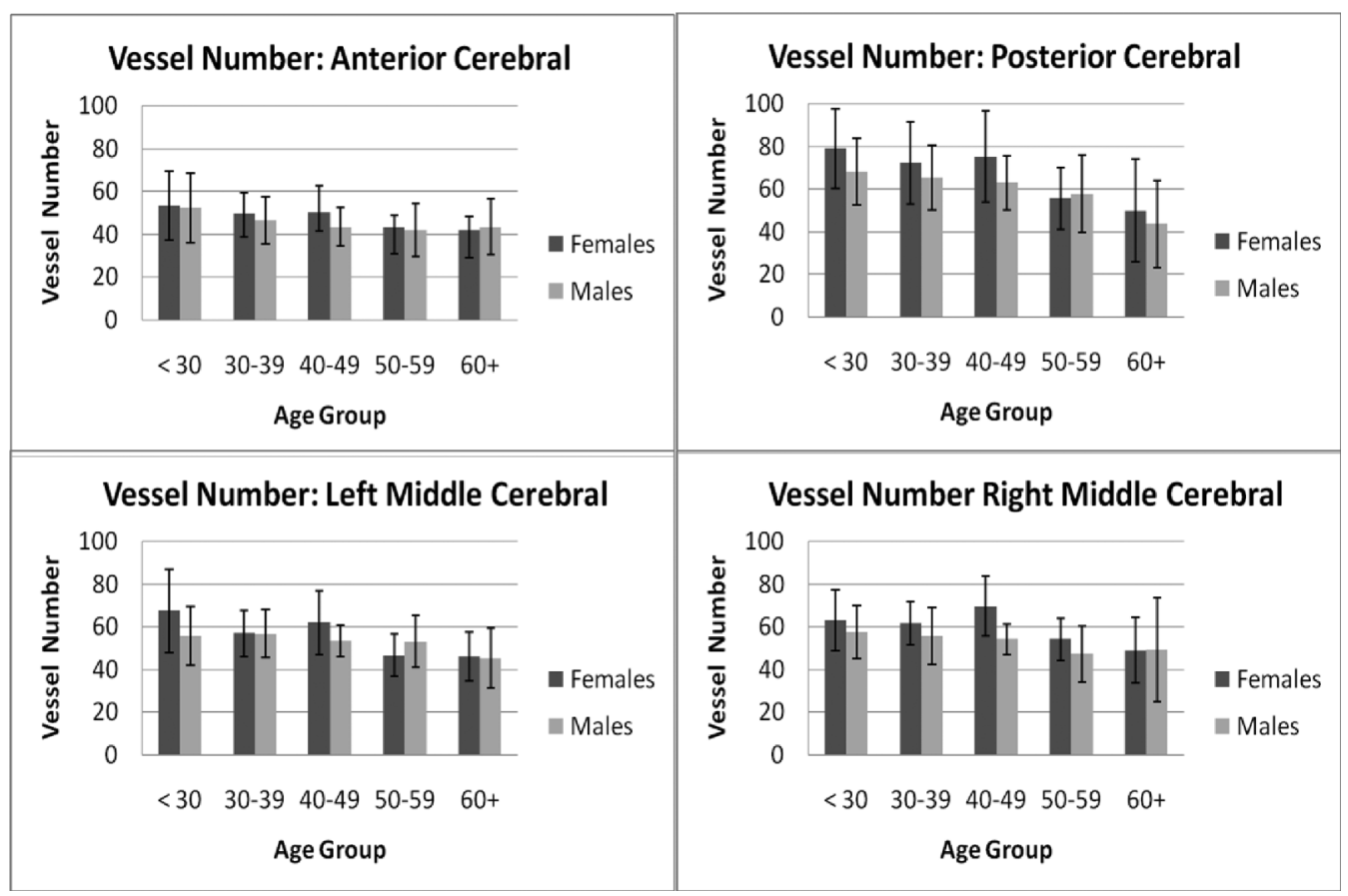

Figure 3.

Means and standard deviations of average vessel number contained within each of the four circulatory groups for men and women in each of the five age groups in years. Vessel number in the anterior cerebral group was less than in the left middle cerebral distribution $(p=0.0032)$; vessel numbers in the left and right middle cerebral distributions did not differ significantly; vessel numbers in the posterior circulation were significantly greater than in any other distribution $(\mathrm{p}<0.001)$. Both genders exhibited a trend toward vessel loss with age in all 4 anatomical regions. The vessel count in the left middle cerebral circulation was significantly lower in subjects aged 60+ than in those aged 18-29 $(\mathrm{p}=0.002)$. Results were most marked in the posterior circulation, for which the vessel count was significantly lower in those aged 60+ than in any other age group (all p-values less than 0.0025 ). 


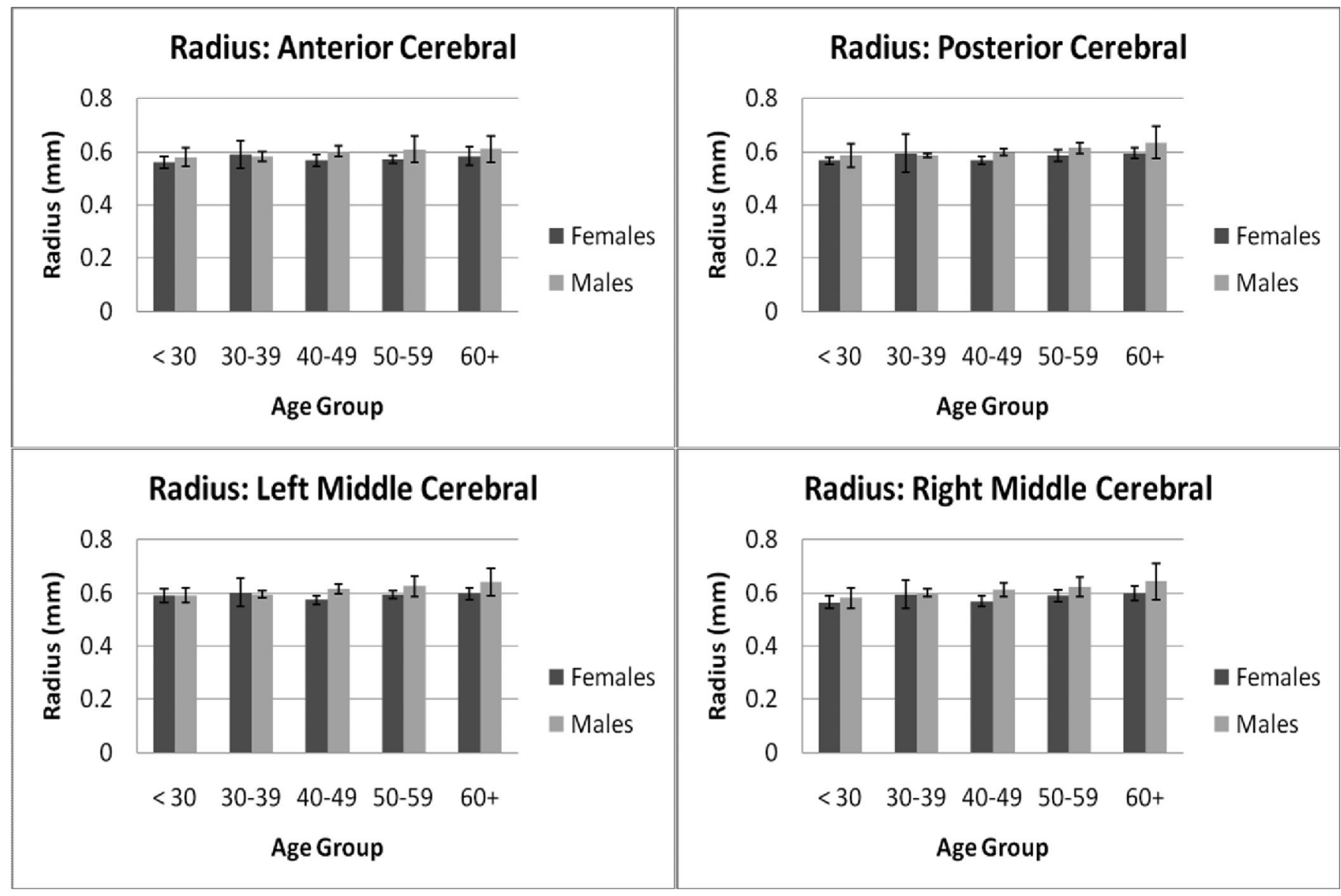

Figure 4.

Means and standard deviations of average radius in $\mathrm{mm}$ for all vessels contained within each of the four circulatory groups for men and women in each of the five age groups. In all four anatomical regions, males exhibited vessels of larger average radius than did women (anterior cerebral group. $\mathrm{p}=0.0021$; left middle cerebral group, $\mathrm{p}=0.0006$; right middle cerebral group, $\mathrm{p}=0.0002$; posterior cerebral group, $\mathrm{p}=0.0033$ ). Aging was also associated with increase in average radius. For those aged $60+$, the average radius of the right middle cerebral group was larger than that of subjects aged $18-29(\mathrm{p}=0.0008)$, as was the average radius of the posterior cerebral circulation $(\mathrm{p}=0.0058)$. No significant interaction between gender and age was found. 


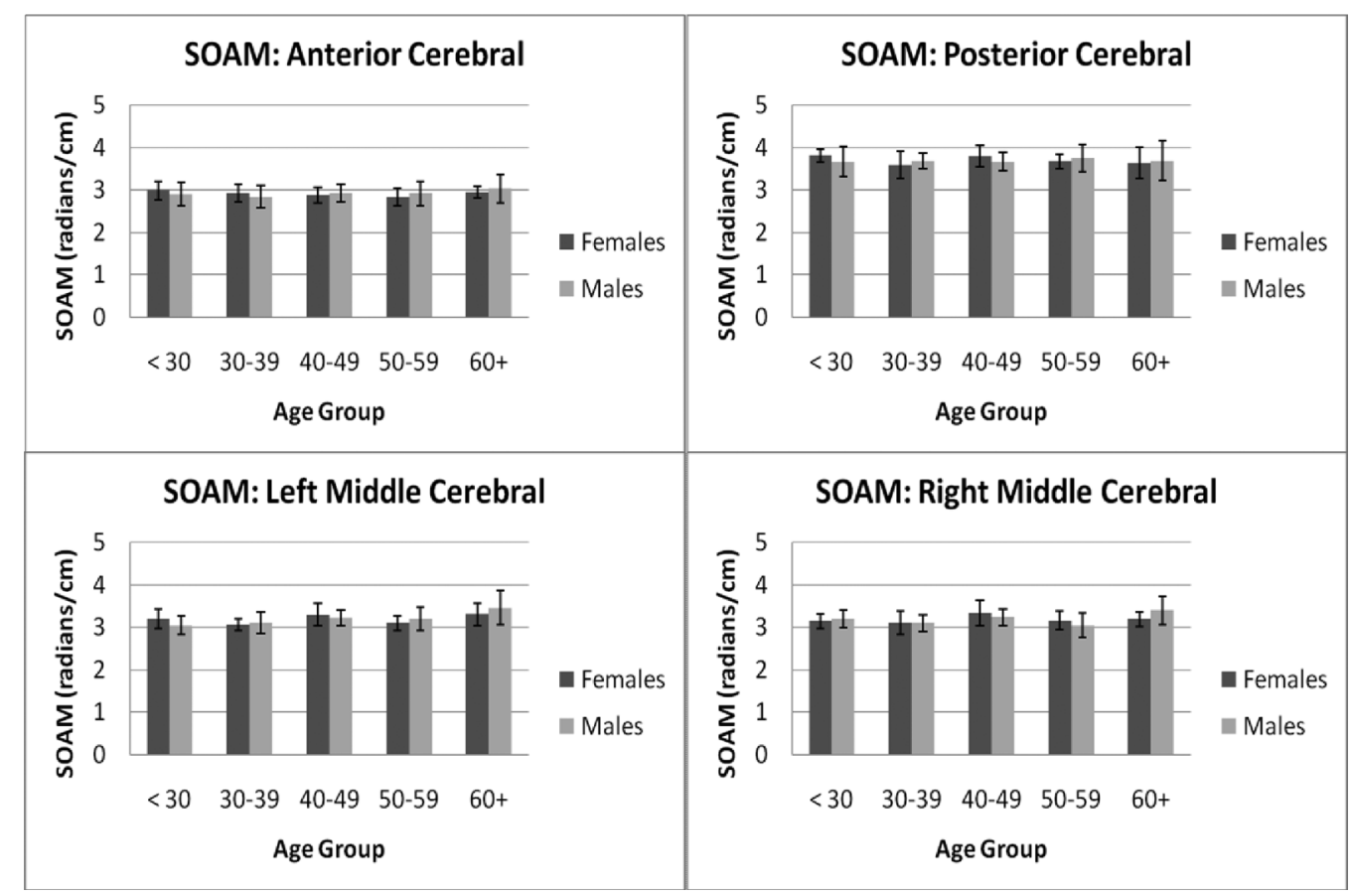

Figure 5.

Means and standard deviations of the tortuosity measure SOAM in radians/cm for all vessels contained within each of the four circulatory groups as calculated for men and women in each of the five age groups. SOAM values differed significantly across anatomical regions $(\mathrm{p}<0.0001)$. The posterior circulation possessed the highest values, the anterior cerebral SOAM was less than the left middle cerebral SOAM, and no significant differences were observed between the left and right middle cerebral SOAMs. Significant changes with aging were limited to the left middle cerebral distribution. Subjects aged 60+ exhibited a significant increase in tortuosity by SOAM compared to those 18-29 $(\mathrm{p}=0.0118)$ and 30-39 $(\mathrm{p}=0.0025)$. 


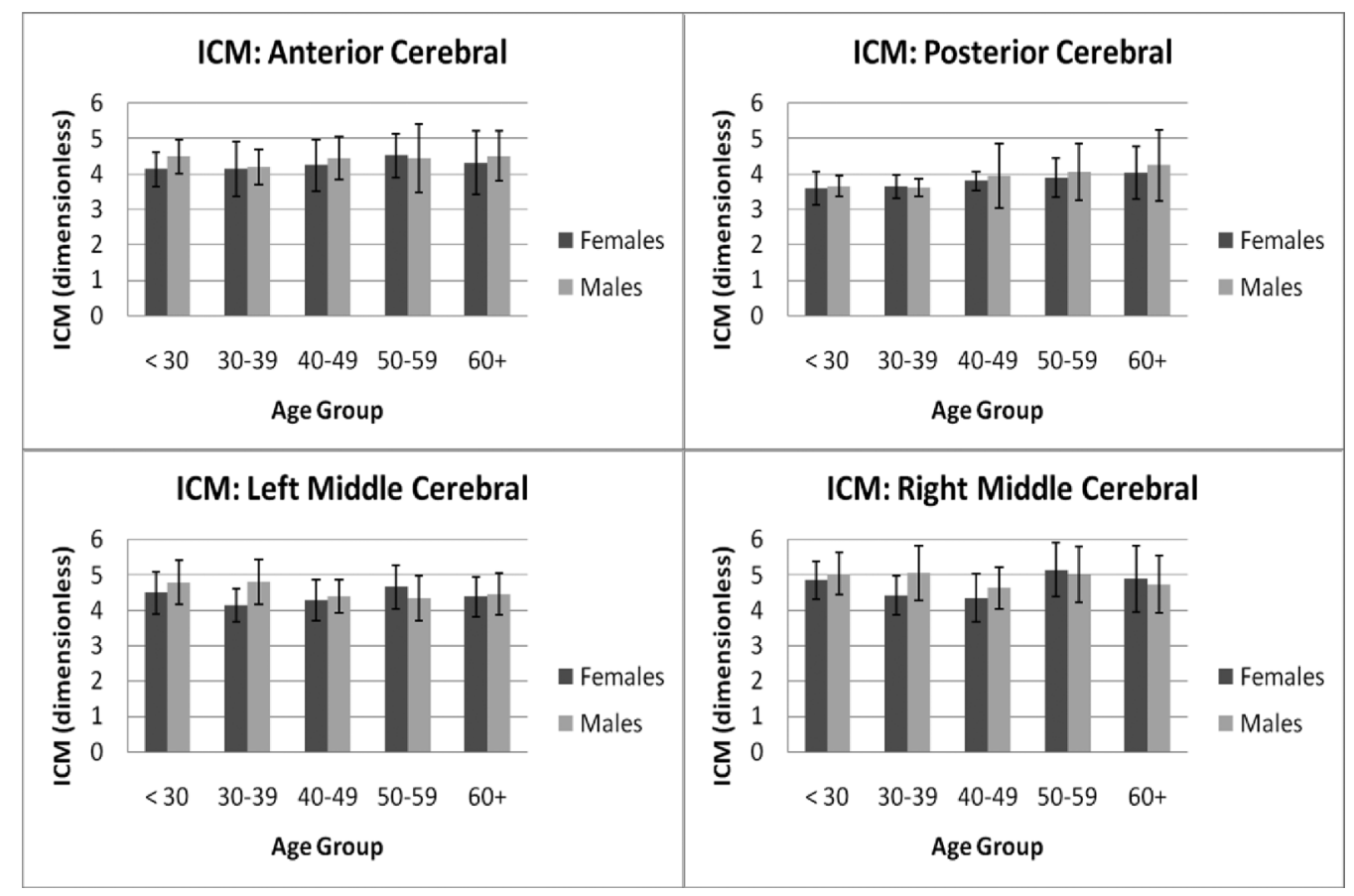

Figure 6.

Means and standard deviations of the tortuosity measure ICM in dimensionless numbers for all vessels contained within each of the four circulatory groups as calculated for men and women in each of the five age groups. ICM values varied significantly by anatomical region; the left middle cerebral circulation had significantly smaller ICM than the right middle cerebral circulation $(\mathrm{p}=0.002)$ and significantly larger ICM than the posterior circulation $(\mathrm{p}<0.0001)$. No significant difference was detected between the anterior and left middle cerebral groups. No significant differences were detected by gender or by age in any anatomical region. 


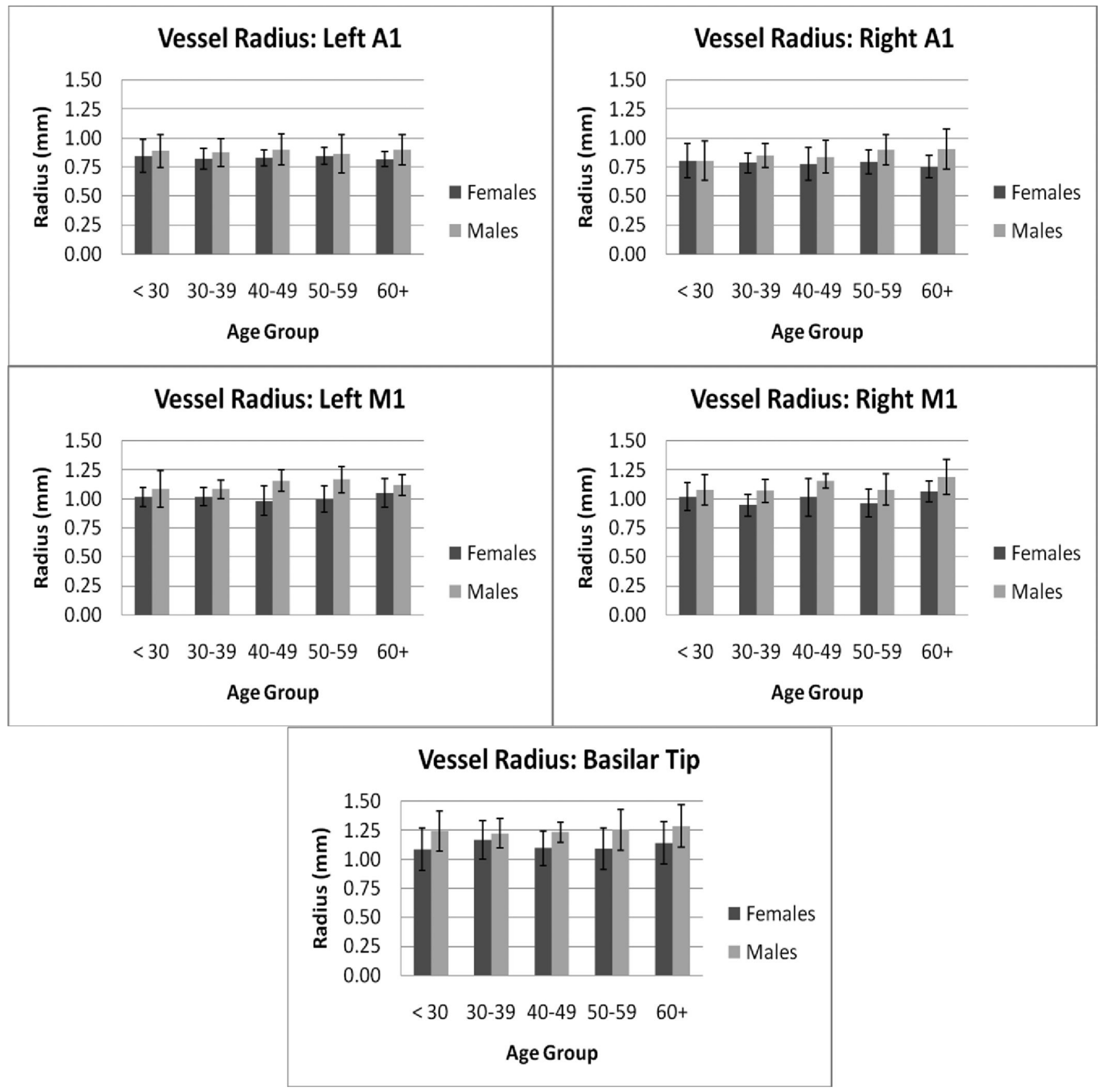

Figure 7.

Means and standard deviations of the radius in $\mathrm{mm}$ for five individual, major named vessels as calculated for both men and women in each of the five age groups. Males consistently displayed higher average vessel radii than females across all anatomical regions and all age groups (p-values all less than 0.02). Older age groups tended to display larger vessel radii with the difference most consistently present in the right M1 arterial segment $(\mathrm{p}=0.015)$; for the right $\mathrm{M} 1$, age group 1 possessed smaller vessel radii than age group $5(\mathrm{p}=0.042)$; age group2 had smaller vessel radii than age group $3(\mathrm{p}=0.047)$; age group 2 had smaller vessel radii than age group $5(\mathrm{p}=0.002)$; age group 4 had smaller vessel radii than age group $5(\mathrm{p}=0.007$.) 


\section{Normalized Vessel Count}

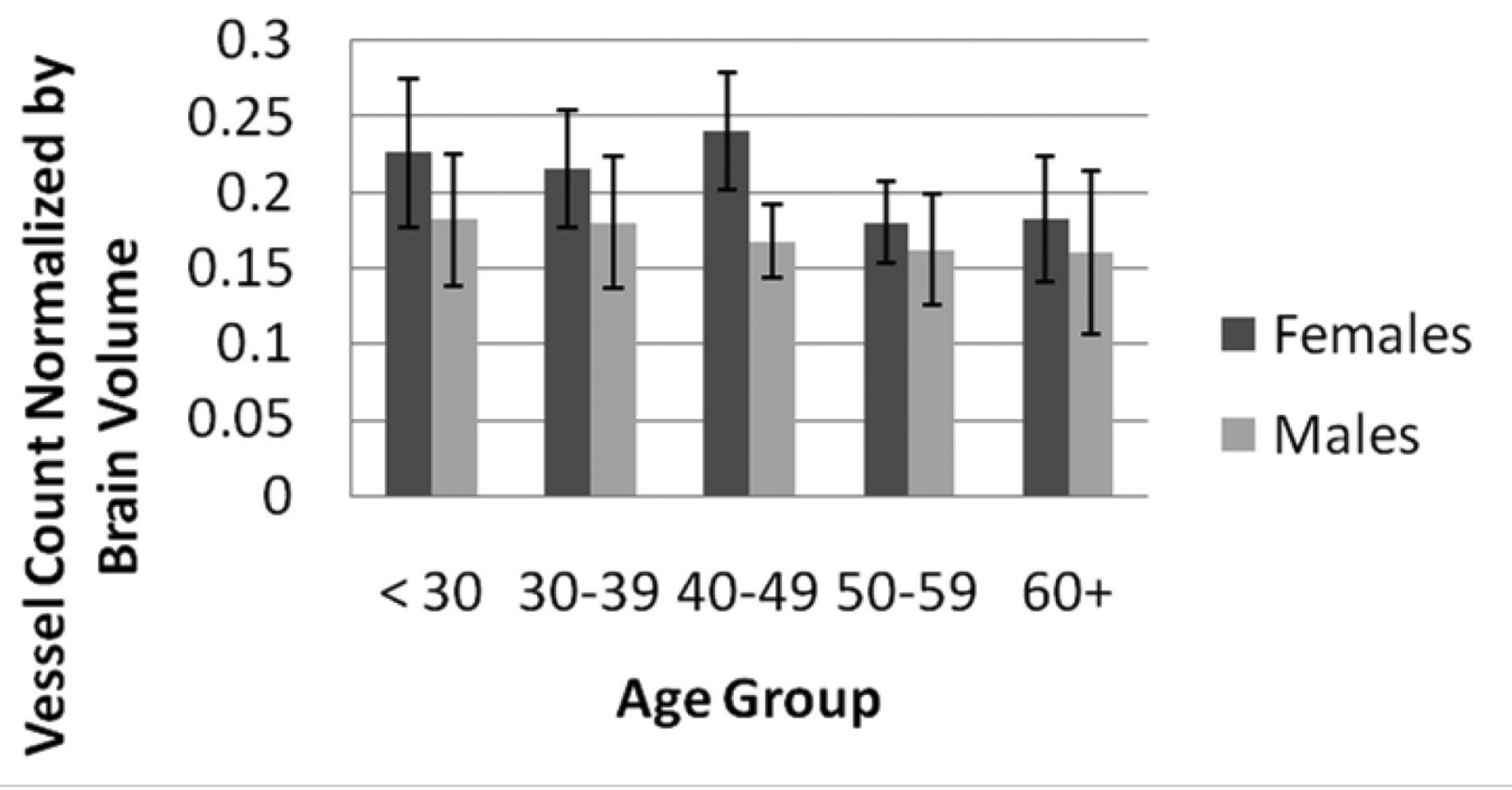

Figure 8.

Total vessel count normalized by brain volume as calculated for both men and women over five age groups. Females had higher vessel counts than males $((p<0.0001)$ and the three younger age groups had higher vessel counts than the two older groups $(p=0.0005)$. 